\title{
Neutrosophic C-means clustering with local information and noise distance-based kernel metric image segmentation
}

\author{
Zhenyu Lu ${ }^{\mathrm{a}, \mathrm{b}}$, Yunan $\mathrm{Qiu}^{\mathrm{a}}$, Tianming Zhan ${ }^{\mathrm{c}, *}$ \\ a School of Electronic and Information Engineering, Nanjing University of Information Science and Technology, Jiangsu, Nanjing, China \\ bJiangsu Collaborative Innovation Center on Atmospheric Environment and Equipment, Jiangsu, Nanjing, China \\ 'School of Information and Engineering, Nanjing Audit University, Jiangsu, Nanjing, China
}

\section{A R T I C L E I N F O}

\section{Article history:}

Received 15 July 2018

Revised 17 November 2018

Accepted 29 November 2018

Available online 1 December 2018

\section{Keywords:}

Image segmentation

Noise clustering

Fuzzy clustering

Nutrosophic clustering

\begin{abstract}
A B S T R A C T
The traditional FCM algorithm is developed on the basis of classical fuzzy theory, though the classical fuzzy theory has its own limitations. The lack of expressive ability of uncertain information makes it hard for FCM algorithm to handle clustered boundary pixels and outliers. This paper proposes a Neutrosophic C-means Clustering with local information and noise distance-based kernel metric for image segmentation (NKWNLICM). At first, noisy distance and fuzzy spatial information are introduced to NCM model to improve the robustness of noise image segmentation. Then, the kernel function is used to measure the distance between pixels. By mapping low-dimensional data into high-dimensional data, the classification performance is further improved. At last, the fuzzy factor is redefined based on the distance between the center pixel and its neighborhood. The new fuzzy factor can excellently reflect the influence of neighborhood pixels on central pixels and improve the classification accuracy much better. The experimental results on Berkeley Segmentation Database demonstrates the excellent performance of the proposed method for noisy image segmentation.
\end{abstract}

(c) 2018 Elsevier Inc. All rights reserved.

\section{Introduction}

The traditional FCM algorithm is developed on the basis of fuzzy theory and is widely applied on computer version [1-3]. However, the fuzzy theory has certain limitations [4]. The lack of expressive ability of uncertain information makes the FCM algorithm unable to handle clustered boundary pixels and outliers when segmenting the image. The traditional FCM algorithm only considers the pixel's gray information when performing image segmentation, and ignores the spatial neighborhood information of the image, which makes the algorithm very sensitive to noise and isolated points [5]. When it is used to process images with noises, the obtained results will suffer from a lower quality [6]. Due to above drawbacks of the FCM algorithm, Dave [7] proposed a noise clustering algorithm (NC), which uses a subset of parameters to represent noise class based on the FCM algorithm. The algorithm reduces the sideeffect of noises on the final clustering results to some extent. Krinidis et al. [8] proposed a fuzzy local information C-means algorithm (FLICM), which used a fuzzy local information to associate local spatial information with local gray information. The algorithm

\footnotetext{
This article is part of the Special Issue on Multimodal_Cooperation.

* Corresponding author.

E-mail address: ztm@nau.edu.cn (T. Zhan).
}

improves the robustness in the algorithm to noise data processing. In order to improve the segmentation performance of the FLICM algorithm, Guo [9] improved the fuzzy local information by using pixel neighborhood variance information and replace the Euclidean distance with kernel distance. Fuzzy C-means clustering with local information and kernel metric for image segmentation (KWFLICM) is obtained with strong robustness and noise immunity. Guo et al. [10] improved the FCM on the basis of the Neutrosophic theory, and proposed the Neutrosophic c-means clustering algorithm (NCM). The algorithm not only includes the degree of membership, but also contains the uncertainty and opposition. The FCM algorithm makes the classification of the boundary region more obvious, and overcome the noise effects. Jian et al. [11] proposed a novel framework for underwater image saliency detection by exploiting Quaternionic Distance Based Weber Descriptor (QDWD), pattern distinctness, and local contrast. The algorithm incorporates quaternion number system and principal components analysis (PCA) simultaneously, so as to achieve superior performance. Jing et al. [12] proposed a transductive low-rank multi-view regression (TLRMVR), and it is capable of boosting the performance of micro-video popularity prediction by jointly considering the intrinsic representations of the source and target samples. Zhu et al. [13] propose a novel unsupervised visual hashing approach called semantic-assisted visual hashing (SAVH). 
Its core idea is to effectively extract the rich semantics latently embedded in auxiliary texts of images to boost the effectiveness of visual hashing without any explicit semantic labels. Zhu et al. [14] propose a novel hashing scheme, named as canonical view based discrete multimodal hashing (CV-DMH), to handle high bandwidth consumption of query transmission and the huge visual variations of query images sent from mobile devices. To solve the problem of the lack of the use of learning mechanism in feature representation, Jing et al. [15] propose a joint low-rank and sparse regression (JLRSR) framework to jointly learn a low-rank projection matrix that enables to decompose the original data into a component part and an error part and a sparse regression coefficient vector for image memorability prediction.

From above analysis, Neutrosophic C-means Clustering Algorithm (NCM) which use neutrosophic theory to improve the traditional FCM is effective to handle the boundary pixels and outliers during clustering. However, without any spatial information involved in NCM model, it cannot obtain accurate image segmentation results. Aiming to solve this problem, we use the spatial distribution of the neighborhood and noise distance-based kernel metric term to improve NCM method. At first, noisy distance and fuzzy spatial information are introduced to NCM model to improve the robustness of noise image segmentation. Then, the kernel function is used to measure the distance between pixels. By mapping low-dimensional data into high-dimensional data, the classification performance is further improved. At last, the fuzzy factor is redefined based on the distance between the center pixel and its neighborhood. The new fuzzy factor can excellently reflect the influence of neighborhood pixels on central pixels and improve the classification accuracy much better. The experimental results on Berkeley Segmentation Database demonstrates the excellent performance of the proposed method for noisy image segmentation.

\section{FCM algorithm}

The fuzzy C-means algorithm (FCM) was proposed by Dunn and Bezdek. The core idea is to find the minimum value of the objective function by finding the appropriate membership and clustering center:

$J_{m}(U, V)=\sum_{i=1}^{n} \sum_{j=1}^{C} \mu_{i j}^{m} d_{i j}^{2}\left(x_{i}, v_{j}\right)$

$\mu_{i j}=\left(\sum_{r=1}^{c}\left[\frac{d_{i j}}{d_{i r}}\right]^{\frac{2}{m-1}}\right)^{-1}$

$v_{j}=\frac{\sum_{i=1}^{n} \mu_{i j}^{m} x_{i}}{\sum_{i=1}^{n} \mu_{i j}^{m}}$

where $J(U, V)$ denotes the square sum of the weighted distance from the pixel to the cluster center of the region, $U=\left(\mu_{i j}\right)_{n \times c}$ denotes the degree of membership matrix, $\mathrm{C}$ is the number of clusters of the image, $\mu_{i j}$ is the value of the sample point $x_{i}$ belonging to $j^{\text {th }}$ Class. m represents a fuzzy exponent, at a typical value of 2 . When $\mathrm{m}=1$, the fuzzy clustering degenerates to hard clustering (HCM), $V=\left(v_{1}, v_{2}, \ldots, v_{c}\right)$ is the matrix of clustering center values, $v_{j}$ Represents $j^{\text {th }}$ Cluster Center, and $d_{i j}^{2}\left(x_{i}, v_{j}\right)=\left\|x_{i}-v_{j}\right\|$ represents the Euclidean distance between sample point $x_{i}$ and the cluster center $v_{j}$.

The algorithm first determines the number of clustering and initialize the membership matrix. Then the Clustering Center and Membership matrix are updated repeatedly through formula (2) and (3). When the objective function is less than a certain threshold, all kinds of clustering centers and membership degrees are obtained.

The algorithm has the following disadvantages: Being sensitive to the initial value and having largely dependence on the initial clustering. If the initial cluster center is far away from the global optimal Clustering center, the algorithm is easy to fall into local minima. Meanwhile, it's easy to be disturbed by noise signal, and the segmentation of noisy image is poor.

\section{Noise clusters algorithm}

The noise clustering algorithm (NC) [7] considers noises as an independent class. It regards the noise distance $\delta$, representing the distance between the sample point and the center of the noise cluster, as a constant. It is a key parameter that is critical to the performance of noise clustering. Based on this argument, a simplified statistical average is used to calculate $\delta$ [7];

$\delta^{2}=\lambda \frac{\sum_{i=1}^{N} \sum_{k=1}^{c} d_{i k}^{2}}{N c}$

where $\lambda$ is a noise multiplier used to adjust the effect of noise distance on the algorithm; Nindicates the total number of sample points; crepresents the number of sample clusters; $d_{i k}$ represents the Euclidean distance between the samplex $x_{i}$ and the cluster center $v_{k}$

In the NC algorithm, $u_{* k}$ is used to indicate the degree of membership of the pixel existing in the noise class. The mathematical expression is shown as follows:

$$
u_{* k}=1-\sum_{k=1}^{c} u_{i k}, \quad \forall i \in\{1,2,3, \ldots, N\}
$$

The NC algorithm changes the membership degree constraints based on the FCM algorithm and introduces the noise distance. Its clustering target expression [9] is expressed as follows:

$J(U, V)=\sum_{i=1}^{N} \sum_{k=1}^{C} u_{i k}^{m} d^{2}\left(x_{i}, v_{k}\right)+\sum_{i=1}^{N} \delta^{2}\left(1-\sum_{k=1}^{C} u_{i k}\right)^{m}$

where $U=\left\{u_{i k}\right\}_{c \times N}$ denotes a fuzzy membership matrix; $V=\left\{v_{k}\right\}_{c \times 1}$ denotes a cluster center matrix; $N$ denotes the total number of sample points; $c$ denotes a number of sample clusters; $x_{i}(i=1,2,3, \ldots, N)$ denotes a sample set; $u_{i k}$ indicates a degree of membership of the $i^{\text {th }}$ sample $x_{i}$ belonging to the $k^{\text {th }}$ class area; $v_{k}(k=1,2,3, \ldots, c)$ denotes the $k^{\text {th }}$ cluster center; $d\left(x_{i}, v_{k}\right)$ is the Euclidean distance between the sample $x_{i}$ and the cluster center $v_{k} ; m \in[1,+\infty]$ is the fuzzy weighted index, which is usually specified as 2 ; and, $\delta^{2}$ is the noise distance.

Due to the effect of noise distance in the algorithm, the noise clustering algorithm is robust and can get better results when dealing with noisy data. Therefore, the concept of noise distance can be combined with other clustering algorithms to improve the robustness of the algorithm.

\section{Neutrosophic C-means cluster}

\subsection{Neutrosophic theory}

In order to address those limitations of the classical fuzzy theory [4] and improve its capability of processing and expressing uncertain information, Smarandache [16] proposed the Neutrosophic theory, which is a generalization of other extended theories. The Neutrosophic theory can not only represent non-deterministic issues in a better way, but also work out the unsolved problems when applying the fuzzy theory.

The basic idea of the Neutrosophic theory is that any viewpoint has a degree of truth, uncertainty, and falsity. Hence, $T$, I, and Fhave been introduced as Neutrosophic Components, which represent 
the authenticity, uncertainty, and absurdity of events respectively. These neutral elements are named true, indeterminate and false values.

\subsection{Neutrosophic C-means clustering algorithm}

In cluster analysis, traditional fuzzy clustering methods can only describe the degree of every group. In fact, especial for the samples on the boundary region between different groups, it is difficult to determine which group they belong to and what partitions they join in. In order to solve these problems, Guo et al. [10] improved the FCM on the basis of the Neutrosophic theory, and proposed the Neutrosophic C-means clustering algorithm (NCM) [10]. A new unique set $A$ has been proposed, which regards as the union of the determinant clusters and indeterminate clusters. Let $A=C_{j} \cup B \cup R, j=1,2, \ldots, c$ where $C_{j}$ is an indeterminate cluster, $B$ regards the clusters in boundary regions, $R$ is associated with noisy data and $\cup$ is the union operation. $B$ and $R$ are two kinds of indeterminate clusters. $T$ is defined as the degree to determinant clusters, $I$ is the degree to the boundary clusters, and $F$ is the degree belonging to the noisy data set. Considering the clustering with indeterminacy, a new objective function and membership are defined as:

$$
\begin{aligned}
& J(T, I, F, C)=\sum_{i=1}^{N} \sum_{k=1}^{C}\left(w_{1} T_{i k}\right)^{m}\left\|x_{i}-v_{k}\right\|^{2} \\
& +\sum_{i=1}^{N} \sum_{k=1}^{\left(\begin{array}{l}
c \\
2
\end{array}\right)}\left(w_{2} I_{2 i k}\right)^{m}\left\|x_{i}-\overline{v_{2 k}}\right\|^{2} \\
& +\sum_{i=1}^{N} \sum_{k=1}^{\left(\begin{array}{c}
c \\
3
\end{array}\right)}\left(w_{3} I_{3 i k}\right)^{m}\left\|x_{i}-\overline{v_{3 k}}\right\|^{2} \\
& +\sum_{i=1}^{N} \sum_{k=1}^{\left(\begin{array}{c}
c \\
4
\end{array}\right)}\left(w_{4} I_{4 i k}\right)^{m}\left\|x_{i}-\overline{v_{4 k}}\right\|^{2}+\ldots \\
& +\sum_{i=1}^{N} \sum_{k=1}^{\left(\begin{array}{c}
c \\
c
\end{array}\right)}\left(w_{c} I_{c i k}\right)^{m}\left\|x_{i}-\overline{c_{c k}}\right\|^{2}+\sum_{i=1}^{N}\left(\overline{w_{c+1}} F_{i}\right)^{m}
\end{aligned}
$$

where $w_{i}$ is the weight factor. $\delta$ is used to control the number of objects considered as outliers. When the clustering number $C$ is greater than 3 , the objective function is very complex and time consuming. After simplification, the objective function is rewritten as:

$$
\begin{aligned}
J(T, I, F, C)= & \sum_{k=1}^{C}\left(w_{1} T_{i k}\right)^{m}\left\|x_{i}-v_{k}\right\|^{2} \\
& +\sum_{i}^{N}\left(w_{2} I_{i}\right)^{m}\left\|x_{i}-\overline{v_{\text {imax }}^{2}}\right\|^{2}+\sum_{i}^{N}\left(\overline{w_{c+1}} F_{i}\right)^{2} \delta^{2}
\end{aligned}
$$

where, $\overline{v_{\text {imax }}}=\frac{v_{p_{i}}+v_{q_{i}}}{2}, p_{i}=\underset{k=1,2, \ldots, C}{\operatorname{argmax}}\left(T_{i k}\right)$, and $q_{i}=\underset{k \neq p_{i} \cap k=1,2, \ldots, C}{\operatorname{argmax}}\left(T_{i k}\right)$.

In above equations, $m$ is a constant, and $p_{i}$ and $q_{i}$ are the cluster numbers with the biggest and second biggest value. When the $p_{i}$ and $q_{i}$ are identified, the $\bar{\nu}_{\text {imax }}$ is calculated and its value is a constant number for each data point $i$, and will not change any more. $T_{i k}, I_{i}$ and $F_{i}$ are the membership values belonging to the determinate clusters, boundary regions and noisy data set, $0<T_{i k}, I_{i}, F_{i}<1$ which satisfy with the following formula:

$\sum_{k=1}^{C} T_{i k}+I_{i}+F_{i}=1$

The partitioning is carried out through an iterative optimization of the objective function, and the membership $T_{i k}, I_{i}, F_{i}$ and the cluster centers $v_{k}$ are updated in each iteration. The $v_{\text {imax }}$ is calculated according to indexes of the largest and second largest value of $T_{i k}$ of each iteration. The iteration will sustain until $\max \left\{\left|T_{i k}^{(h+1)}-T_{i k}^{(h)}\right|\right\}<\varepsilon$ or $h \geq H_{\max }$, in which $\varepsilon$ a termination criterion between 0 and 1 is, $\mathrm{k}$ is the iteration step, and $h$ is the number of iterations.

\section{Proposed method}

In NCM algorithm, since the objective function does not involve any spatial information. If we directly use it for image segmentation, the segmentation performance is not very well. In addition, using the maximum membership principle to determine pixel labels may produce some segmentation errors. Therefore, spatial neighborhood information should be added to the objective function to reduce the influence of undesired factors on the final classification result.

Aiming to solve the problems of NCM algorithm, we propose a new clustering algorithm, Neutrosophic C-means clustering with Local Information and Kernel Metric noise distance-based for Image Segmentation (NKWNLICM). The objective function is

$$
\begin{aligned}
& J(T, I, F, C)=\sum_{i=1}^{N} \sum_{k=1}^{c}\left(w_{1} T_{i k}\right)^{m}\left(\left\|\Phi\left(x_{i}\right)-\Phi\left(v_{k}\right)\right\|^{2}+G_{i k}\right) \\
& +\sum_{i=1}^{N} \sum_{l=1}^{\left(\begin{array}{l}
2 \\
c
\end{array}\right)}\left(w_{2} I_{i l}\right)^{m}\left(\left\|\Phi\left(x_{i}\right)-\Phi\left(\overline{v_{l}}\right)\right\|^{2}+\overline{G_{i l}}\right) \\
& +\sum_{i=1}^{N}\left(w_{3} F_{i}\right)^{m} \delta^{2}
\end{aligned}
$$

where $T_{i k}$ denotes the extent to which element $i$ belongs to cluster $k$, $I_{i l}$ denotes the degree to which element $i$ belongs to two cluster boundaries in cluster $c, F_{i}$ denotes the degree to which element $i$ belongs to noise; $\bar{v}_{l}$ is the average of any two classes value. $w_{i}$ is the weighting factor, $\delta$ is the noise distance, $\overline{G_{i l}}$ and $G_{i k}$ are local information.

When the clustering number $C$ is greater than 3 , the objective function in Eq. (10) is very complex and time consuming. In this situation, if we only consider the two closest determinate clusters which have the top two largest membership values, the objective function will be simplified. Meanwhile, computation cost will be reduced without decreasing the clustering accuracy greatly.

$\delta^{2}=\lambda\left[\frac{\sum_{i=1}^{N} \sum_{k=1}^{c}\left\|\Phi\left(x_{i}\right)-\Phi\left(v_{k}\right)\right\|^{2}}{N c}\right]$

$G_{i k}=\sum_{j \in N_{i}, i \neq j} w_{i j}\left(1-T_{j k}\right)^{m}\left\|\Phi\left(x_{i}\right)-\Phi\left(v_{k}\right)\right\|^{2}$

where $T_{i k}, I_{i}$ and $F_{i}$ are the membership values belonging to the determinated clusters, boundary regions and noisy data set, $0<T_{i k}, I_{i}, F_{i}<1$ which satisfy with the following formula:

$\sum_{k=1}^{C} T_{i k}+\sum_{l=1}^{\left(\begin{array}{l}2 \\ C\end{array}\right)} I_{i l}+F_{i}=1$

According to the above formula, the Lagrange objective function is constructed as

$$
\begin{aligned}
& L(T, I, F, c, \lambda)=\sum_{i=1}^{N} \sum_{k=1}^{c}\left(w_{1} T_{i k}\right)^{m}\left(\left\|\Phi\left(x_{i}\right)-\Phi\left(v_{k}\right)\right\|^{2}+G_{i k}\right)
\end{aligned}
$$

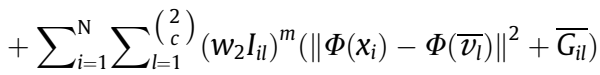

$$
\begin{aligned}
& +\sum_{i=1}^{N}\left(w_{3} F_{i}\right)^{m} \delta^{2} \\
& +\sum_{i=1}^{N} \lambda_{i}\left(\sum_{k=1}^{c} T_{i k}+\sum_{l=1}^{\left(\begin{array}{l}
2 \\
c
\end{array}\right)} I_{i l}+F_{i}-1\right)=0
\end{aligned}
$$

To minimize the Lagrange objective function, we use the following operations:

$$
\begin{aligned}
& \frac{\partial L}{\partial T_{i k}}=m\left(w_{1} T_{i k}\right)^{m-1}\left(\left\|\Phi\left(x_{i}\right)-\Phi\left(v_{k}\right)\right\|^{2}+G_{i k}\right)-\lambda_{i} \\
& \frac{\partial L}{\partial I_{i l}}=m\left(w_{2} I_{i l}\right)^{m-1}\left(\left\|\Phi\left(x_{i}\right)-\Phi\left(\overline{v_{l}}\right)\right\|^{2}+\overline{G_{i l}}\right)-\lambda_{i}
\end{aligned}
$$


$\frac{\partial L}{\partial F_{i}}=m\left(w_{3} F_{i}\right)^{m-1} \delta^{2}-\lambda_{i}$

$\frac{\partial L}{\partial v_{k}}=-2 \sum_{i=1}^{N}\left(w_{1} T_{i k}\right)^{2}\left(\Phi\left(x_{i}\right)-\Phi\left(v_{k}\right)\right)$

Using Euclidean universal numbers, let $\frac{\partial L}{\partial T_{i k}}=0, \frac{\partial L}{\partial I_{i}}=0, \frac{\partial L}{\partial F_{i}}=0$, $\frac{\partial L}{\partial v_{k}}=0$, then

$T_{i j}=\frac{1}{w_{1}}\left(\frac{\lambda_{i}}{m}\right)^{\frac{1}{m-1}}\left(\left\|\Phi\left(x_{i}\right)-\Phi\left(v_{k}\right)\right\|^{2}+G_{i k}\right)^{-\frac{1}{m-1}}$

$I_{i l}=\frac{1}{W_{2}}\left(\frac{\lambda_{i}}{m}\right)^{\frac{1}{m-1}}\left(\left\|\Phi\left(x_{i}\right)-\Phi\left(\overline{v_{l}}\right)\right\|^{2}+\overline{G_{i l}}\right)^{-\frac{1}{m-1}}$

$F_{i}=\frac{1}{W_{3}}\left(\frac{\lambda_{i}}{m}\right)^{\frac{1}{m-1}} \delta^{-\frac{2}{m-1}}$

$v_{k}=\frac{\sum_{i=1}^{N}\left(w_{1} T_{i k}\right)^{m} T_{i k} x_{i}}{\sum_{i=1}^{N}\left(w_{1} T_{i k}\right)^{m} T_{i k}}$

Let $\left(\frac{\lambda_{i}}{m}\right)^{\frac{1}{m-1}}=K_{i}$, then we obtain:

$1=\sum_{j=1}^{c} \frac{K_{i}}{W_{1}}\left(\left\|\Phi\left(x_{i}\right)-\Phi\left(v_{k}\right)\right\|^{2}+G_{i k}\right)^{-\frac{1}{m-1}}$

$+\sum_{l=1}^{\left(\begin{array}{l}2 \\ c\end{array}\right)} \frac{K_{i}}{W_{2}}\left(\left\|\Phi\left(x_{i}\right)-\Phi\left(\overline{v_{l}}\right)\right\|^{2}+\overline{G_{i l}}\right)^{-\frac{1}{m-1}}+\frac{K_{i}}{W_{3}} \delta^{-\frac{2}{m-1}}$

$K_{i}=\left[\frac{1}{w_{1}} \sum_{k=1}^{c}\left(\left\|\Phi\left(x_{i}\right)-\Phi\left(v_{k}\right)\right\|^{2}+G_{i k}\right)^{-\frac{1}{m-1}}\right.$

$\left.+\sum_{l=1}^{\left(\begin{array}{l}2 \\ c\end{array}\right)} \frac{1}{w_{2}}\left(\left\|\Phi\left(x_{i}\right)-\Phi\left(\overline{v_{l}}\right)\right\|^{2}+\overline{G_{i l}}\right)^{-\frac{1}{m-1}}+\frac{1}{w_{3}} \delta^{-\frac{2}{m-1}}\right]^{-1}$

Therefore,

$T_{i k}=\frac{K_{i}}{w_{1}}\left(\left\|\Phi\left(x_{i}\right)-\Phi\left(v_{k}\right)\right\|^{2}+G_{i k}\right)^{-\frac{1}{m-1}}$

$I_{i l}=\frac{K_{i}}{W_{2}}\left(\left\|\Phi\left(x_{i}\right)-\Phi\left(\overline{v_{l}}\right)\right\|^{2}+\overline{G_{i l}}\right)^{-\frac{1}{m-1}}$

$F_{i}=\frac{K_{i}}{W_{3}} \delta^{-\frac{2}{m-1}}$

$\overline{v_{l}}=\frac{1}{2}\left(v_{q}+v_{p}\right), p, q \in\{1,2, \ldots, c\}, p \neq q$

The partitioning is carried out through an iterative optimization of the objective function, and the membership $T_{i k}, I_{i}, F_{i}$ and the cluster centers $v_{k}$ are updated in each iteration. The $v_{\text {imax }}$ is calculated according to indexes of the top two largest value of $T_{i k}$ in each iteration. The iteration will continuous until $\max \left\{\left|T_{i k}^{(h+1)}-T_{i k}^{(h)}\right|\right\}<\varepsilon$ or $h \geq H_{\max }$, where $\varepsilon$ a termination criterion between 0 and $1, \mathrm{k}$ is the iteration step, and $h$ is the number of iterations.

The above equations allow the formulation of NKWNLICM algorithm. It can be summarized in the following steps:

Step 1: Initialize the $m, \varepsilon, w_{1}, w_{2}, w_{3}, H_{\max }$ and $w_{i j}$;

Step 2: Initialize $T^{(0)}, I^{(0)}$ and $F^{(0)}$, let $h=0$;

Step 3: Calculate the centers vectors $v^{(h)}$ and cluster boundary $\overline{v_{i l}}$ at $h$ step using Eq. (22) and Eq. (28);

Step 4: Calculate noise distance using Eq. (11);

Step 5: Update $T^{(h)}$ to $T^{(h+1)}, I^{(h)}$ to $I^{(h+1)}$, and $F^{(h)}$ to $F^{(h+1)}$ using Eqs. (25)-(27):
Table 1

The notation table of main symbols.

\begin{tabular}{|c|c|c|c|}
\hline $\mathrm{J}$ & Weighted distance & $\sigma$ & Noise distance \\
\hline $\mathrm{U}$ & Membership & $\lambda$ & Noise multiplier \\
\hline C & Number of clusters & $\mathrm{N}$ & Total number of sample points \\
\hline $\mathrm{m}$ & Fuzzy exponent & $\mathrm{d}$ & Euclidean distance \\
\hline $\mathrm{V}$ & $\begin{array}{l}\text { Clustering center } \\
\text { values }\end{array}$ & w & Weight factor \\
\hline$\varepsilon$ & $\begin{array}{l}\text { Termination } \\
\text { criterion }\end{array}$ & $\mathrm{T}$ & $\begin{array}{l}\text { Membership values belonging to } \\
\text { determinate clusters }\end{array}$ \\
\hline G & Local information & I & $\begin{array}{l}\text { Membership values belonging to boundary } \\
\text { regions }\end{array}$ \\
\hline h & $\begin{array}{l}\text { Number of } \\
\text { iterations }\end{array}$ & $\mathrm{F}$ & $\begin{array}{l}\text { Membership values belonging to noisy data } \\
\text { set }\end{array}$ \\
\hline
\end{tabular}

Step 6: If $\left|T^{(h+1)}-T^{(h)}\right|<\varepsilon$ or $h \geq H_{\max }$ then stop; otherwise return to Step 3, let $h=h+1$;

Step 7: Assign each data into the class with the largest $T M=[T, I, F]$ value: $x(i) \in h^{\text {th }}$ class if $h=\operatorname{argmax}\left(T M_{i j}\right)$

To easily understand the proposed NKWNLICM, the main symbols are summarized in Table 1.

The effects of each component in the proposed method are given as follows:

a) Neutrosophic C-means clustering: In addition of the membership of pixels in each class, Neutrosophic theory estimates the uncertainty of clustering. Thus, the pixels located in edge region can be accurately categorized.

b) Kernel function: Kernel function is used to measure the distance between pixels, thus mapping the complex non-linear problems in the original low-dimensional space to the highdimensional space.

c) Weighed fuzzy factor: The trade-off weighted fuzzy factor contains both the spatial distance information and gray information of neighborhood pixels, it can better reflect the influence of neighborhood pixels on the central pixel, thus further improving the clustering performance.

d) Noise distance term: Introducing noise distance term in classification criteria, the segmentation of the proposed method is robust to noisy image.

e) Fuzzy local information: By considering the fuzzy spatial distribution in the local region of image, the proposed method is able to distinguish between image boundary pixels and noise during classification and further improve the image segmentation performance.

\section{Evaluation criteria}

The evaluation criteria used in this paper are given as follows:

An entropy-based evaluation function (E), which combines both the layout entropy $\left(H_{r}\right)$ and the expected region entropy $\left(H_{l}\right)$, is often used in measuring the effectiveness of a segmentation method [17].

The entropy for region $R_{j}$ is defined as:

$H\left(R_{j}\right)=-\sum_{m \in V_{j}} \frac{L_{j}(m)}{S_{j}} \log \frac{L_{j}(m)}{S_{j}}$

The expected region entropy of segmentation I is defined as:

$H_{r}(I)=\sum_{j=1}^{c}\left(\frac{S_{j}}{S_{I}}\right) H\left(R_{j}\right)$

The layout entropy is defined as:

$H_{l}(I)=-\sum_{j=1}^{c}\left(\frac{S_{j}}{S_{I}}\right) \log \frac{S_{j}}{S_{I}}$ 
The entropy-based evaluation function $\mathrm{E}$ is defined as:

$E=H_{l}(I)+H_{r}(I)$

where $L_{j}(m)$ denotes to the number of pixels in region $\mathrm{j}$ that have a value of $\mathrm{m}$ for feature (e.g. luminance) in the original image. $S_{j}=\left|R_{j}\right|$ denotes to the area of region $R_{j}$. Maximizing the consistency of pixels in each segmentation region and minimizing the consistency of different regions, the best segmentation result is obtained when $\mathrm{E}$ achieved to the minimum value.

Partition coefficient $V_{p c}$ and partition entropy $V_{p e}$ are proposed by Bezdek et al. [18], which is mainly used to measure the pixel membership of the segmented images. The definition is as follows:

$V_{p c}=\frac{1}{n} \sum_{i=1}^{c} \sum_{j=1}^{n} u_{i j}^{2}$

$V_{p e}=-\frac{1}{n} \sum_{i=1}^{c} \sum_{j=1}^{n} u_{i j} \log u_{i j}$

During image classification, the higher compactness of the object indicates the lower the separation between the objects, which means that the segmentation effect is much better. Therefore, the better the clustering effect means the larger the value of $V_{p c}$ and the smaller the value of $V_{p e}$.

The classification accuracy rate $S A[19]$ is defined as follows:

$S A=\sum_{i=1}^{c} \frac{\operatorname{card}\left(A_{i} \cap C_{i}\right)}{\sum_{j=1}^{c} \operatorname{card}\left(C_{j}\right)}$

where $A_{i}$ denotes the set of pixels of the $i^{\text {th }}$ cluster in the segmentation result, $C_{i}$ denotes the set of pixels of the $i^{\text {th }}$ cluster in the standard segmentation image, card is used to calculate the number of elements in the set. The higher the accuracy indicates the better segmentation result.
Reconstruction error rate $V_{R E}$ proposed by Pedrycz et al. [20] is mainly used to measure the difference between the segmented image and the original image. Its definition is given as follows:

$V_{R E}=\frac{1}{N} \sum_{k=1}^{N}\left\|x_{k}-\overline{x_{k}}\right\|^{2}$

where $x_{i}$ represents the gray value of the $i^{\text {th }}$ pixel of the original image and $\overline{x_{i}}$ represents the gray value of the $i^{\text {th }}$ pixel of the reconstructed image, which is defined as follows:

$\overline{x_{k}}=\sum_{i=1}^{c} u_{i k}^{m} v_{k} / \sum_{i=1}^{c} u_{i k}^{m}$

Among them, $u_{i k}^{m}$ denotes the membership degree of cluster $k$, and $v_{k}$ denotes the clustering center value of cluster $k$. The segmented image is reconstructed according to formula (37). The smaller reconstruction error indicates better segmentation result.

\section{Experimental results}

The testing environment of this experiment in this paper is CPU core $2.5 \mathrm{GHz}$, memory $4 \mathrm{~GB}$, and programmed by Matlab 2016a. In this section, in order to validate the effectiveness of this method in image segmentation, a comparative experiment is conducted with images in Berkeley Segmentation Database. The public benchmark based on Berkeley Segmentation Database consists 200 images with hand-labeled segmentation. We use 4 images (camera image and no. 388016, 15088 and 176039 from Berkeley Segmentation Database) to compare the proposed method with other approaches named FCM, FGFCM, FLICM, NCM and KWFLICM to demonstrate the performance in clustering.

In the experiments, the parameter $m$ has the same meaning to the fuzzification constant in the fuzzy clustering algorithm, and its value usually selected as 2 . We selected $N_{R}=8, \lambda_{s}=3$, and $\lambda_{g}=0.5$. The noise type is $20 \%$ salt and pepper noise. We take different

Table 2

The $\mathrm{E}$ values of the segmentation results obtained by different parameters.

\begin{tabular}{|c|c|c|c|c|c|c|c|c|c|c|c|c|}
\hline & \multicolumn{4}{|l|}{$\mathrm{m}$} & \multicolumn{4}{|l|}{$\mathrm{T}$} & \multicolumn{4}{|l|}{$\lambda$} \\
\hline & 2 & 3 & 4 & 5 & 0.8 & 0.6 & 0.4 & 0.2 & 0.1 & 0.2 & 0.5 & 1.0 \\
\hline $\mathrm{Hr}$ & 1.66 & 1.68 & 1.72 & 1.78 & 1.66 & 1.68 & 1.72 & 1.7 & 1.66 & 1.71 & 1.68 & 1.72 \\
\hline $\mathrm{H}_{l}$ & 0.36 & 0.39 & 0.38 & 0.37 & 0.36 & 0.36 & 0.36 & 0.37 & 0.36 & 0.36 & 0.37 & 0.36 \\
\hline $\mathrm{E}$ & 2.02 & 2.08 & 2.13 & 2.15 & 2.02 & 2.05 & 2.06 & 2.05 & 2.02 & 2.02 & 2.14 & 2.10 \\
\hline
\end{tabular}

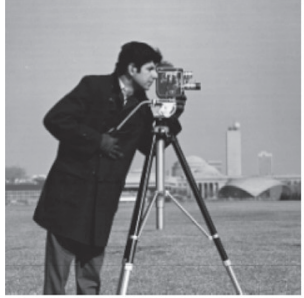

(a) The original image

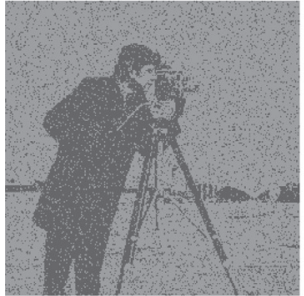

(e) FLICM

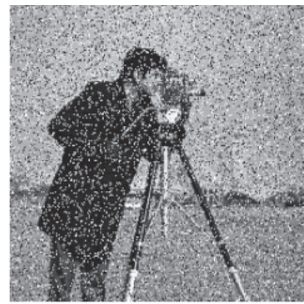

(b) Noise image

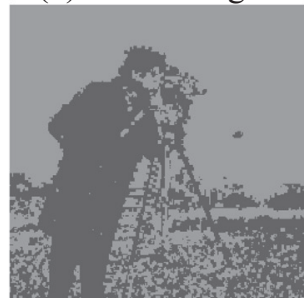

(f) NCM

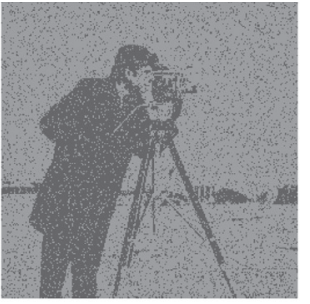

(c) FCM

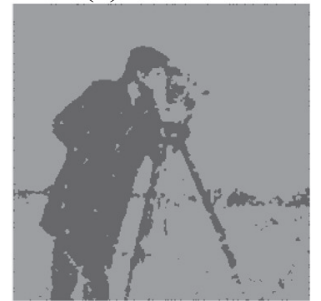

(g) KWFLICM

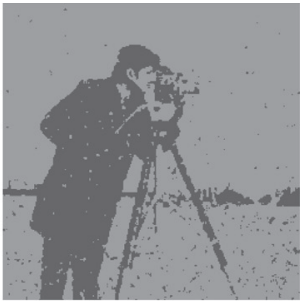

(d) FGFCM

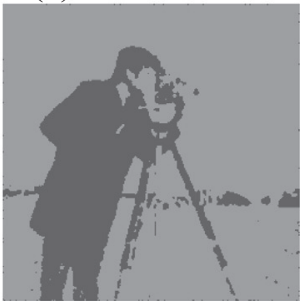

(h) NKWNLICM

Fig. 1. Comparison of camera image segmentation results. 
values for each parameter to select the best segmentation parameter settings. It can be seen from Table 2 that when $\mathrm{m}$ takes $2, \mathrm{~T}$ takes 0.8 , and $\lambda$ takes 0.1 , it works best.

We compare the proposed method with other approaches named FCM, FGFCM, FLICM, NCM and KWFLICM to demonstrate their segmentation performance. The original images and segmentation results are shown from Fig. 1 to Fig. 4. Figs. 1(a), 2(a), 3(a), 4 (a) are original images without noise. Figs. 1(b), 2(b), 3(b), 4(b) are images with 20\% salt and pepper noise. Figs. 1(c), 2(c), 3(c), 4(c) are segmentation results of FCM method. Figs. 1(d), 2(d), 3(d), 4(d) are

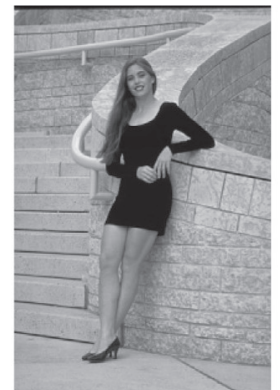

(a) The original image

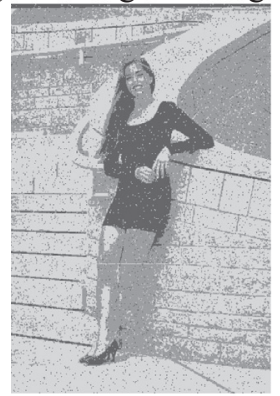

(e) FLICM

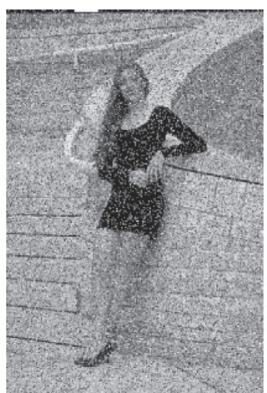

(b) Noise image

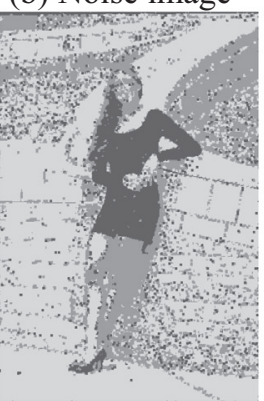

(f) $\mathrm{NCM}$ segmentation results of FGFCM method. Figs. 1(e), 2(e), 3(e), 4(e) are segmentation results of FLICM method. Figs. 1(f), 2(f), 3(f), 4 (f) are segmentation results of NCM method. Figs. 1(g), 2(g), 3(g), $4(\mathrm{~g})$ are segmentation results of KWFLICM method. Figs. 1(h), 2 (h), 3(h), 4(h) are segmentation results of NKWNLICM method. By comparison, it can be clearly seen that the NKWNLICM method has the best segmentation effect, and noise is significantly less than other methods.

For quantitative comparison, the evaluation criteria Vpc, Vpe, $\mathrm{V}_{\mathrm{RE}}, \mathrm{SA}$ and $\mathrm{E}$ are calculated for the segmentation results obtained

Fig. 2. Berkeley University image library 388,016 image segmentation results.

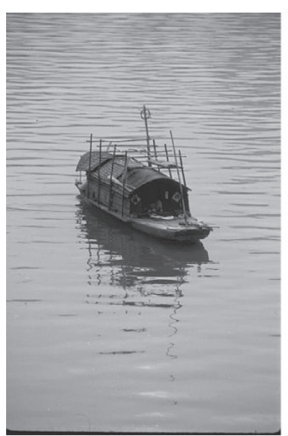

(a) The original image

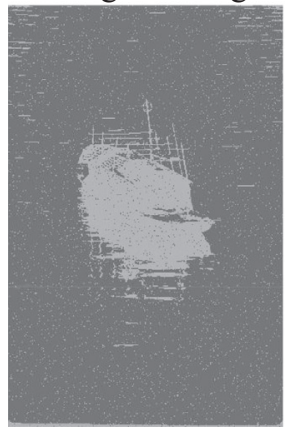

(e) FLICM

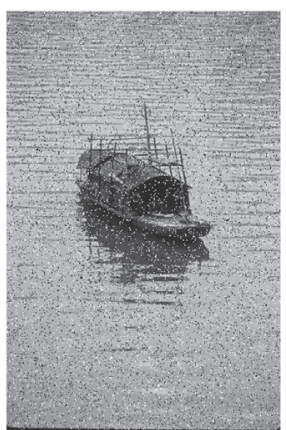

(b) Noise image

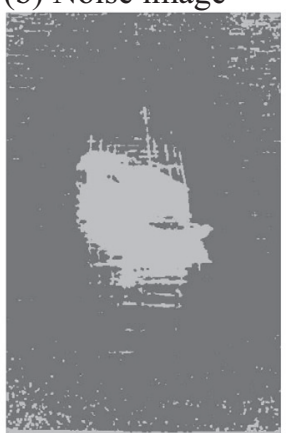

(f) NCM

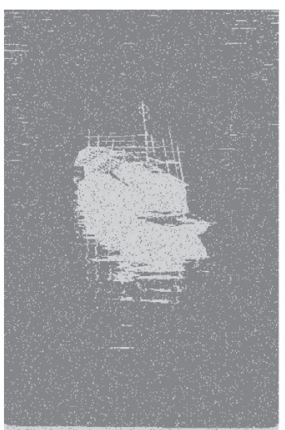

(c) FCM

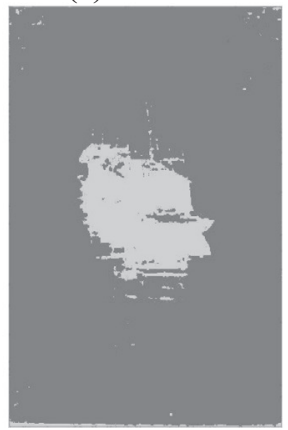

(g) KWFLICM

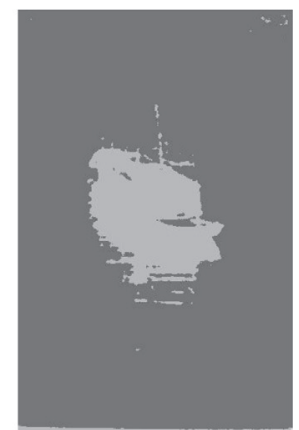

(d) FGFCM

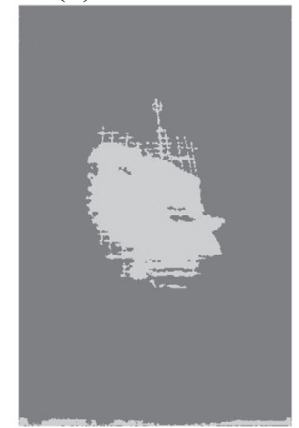

(h) NKWNLICM

Fig. 3. Berkeley University image library 15,088 image segmentation results. 


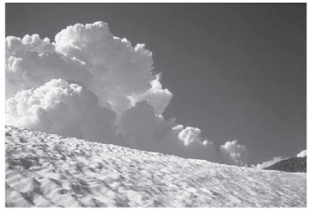

(a) The original image

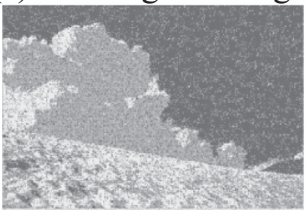

(e) FLICM

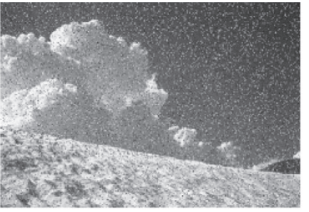

(b) Noise image

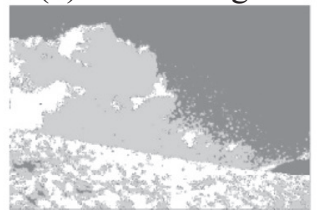

(f) NCM

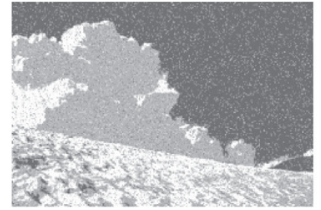

(c) FCM

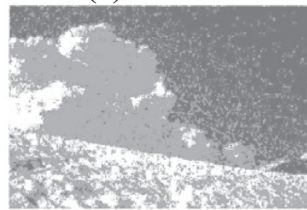

(g) KWFLICM

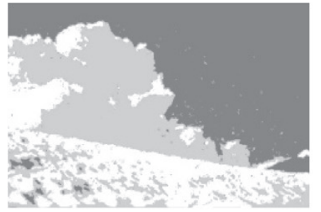

(d) FGFCM

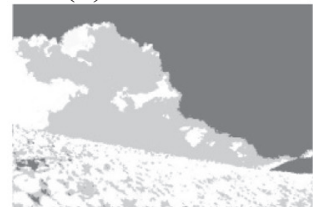

(h) NKWNLICM

Fig. 4. Berkeley University image library 176,039 image segmentation results.

Table 3

The Vpc, Vpe, $V_{\mathrm{RE}}$ and SA values of the segmentation result obtained by different methods on Berkeley Segmentation Database.

\begin{tabular}{|c|c|c|c|c|c|c|}
\hline & FCM & FGFCM & FLICM & $\mathrm{NCM}$ & KWFLICM & NKWNLICM \\
\hline Vpc & 0.9220 & 0.9255 & 0.9303 & 0.9289 & 0.9362 & 0.9962 \\
\hline Vpe & 0.1426 & 0.1269 & 0.1453 & 0.1343 & 0.0998 & 0.0084 \\
\hline $\mathrm{V}_{\mathrm{RE}}$ & 153.48 & 152.06 & 152.71 & 150.63 & 122.71 & 102.49 \\
\hline SA & 90.8362 & 95.2290 & 93.1943 & 93.8031 & 96.1574 & 97.1244 \\
\hline
\end{tabular}

Table 4

The $\mathrm{E}$ of the segmentation result obtained by different methods on each image.

\begin{tabular}{|c|c|c|c|c|c|c|c|}
\hline Image & Metric & FCM & FGFCM & FLICM & NCM & KWFLICM & NKWNLICM \\
\hline Fig. 1 & $\begin{array}{l}\mathrm{H}_{r} \\
\mathrm{H}_{l} \\
\mathrm{E}\end{array}$ & $\begin{array}{l}1.7306 \\
0.4637 \\
2.1943\end{array}$ & $\begin{array}{l}1.7076 \\
0.4576 \\
2.1652\end{array}$ & $\begin{array}{l}1.7248 \\
0.4625 \\
2.1873\end{array}$ & $\begin{array}{l}1.7249 \\
0.4536 \\
2.1785\end{array}$ & $\begin{array}{l}1.7072 \\
0.4566 \\
2.1638\end{array}$ & $\begin{array}{l}1.7026 \\
0.4548 \\
\mathbf{2 . 1 5 7 4}\end{array}$ \\
\hline Fig. 2 & $\begin{array}{l}\mathrm{H}_{r} \\
\mathrm{H}_{l} \\
\mathrm{E}\end{array}$ & $\begin{array}{l}1.8981 \\
0.5176 \\
2.4157\end{array}$ & $\begin{array}{l}1.8813 \\
0.5057 \\
2.3870\end{array}$ & $\begin{array}{l}1.8799 \\
0.5064 \\
2.3863\end{array}$ & $\begin{array}{l}1.8932 \\
0.5184 \\
2.4116\end{array}$ & $\begin{array}{l}1.8792 \\
0.5062 \\
2.3854\end{array}$ & $\begin{array}{l}1.8698 \\
0.4985 \\
\mathbf{2 . 3 6 8 3}\end{array}$ \\
\hline Fig. 3 & $\begin{array}{l}\mathrm{H}_{r} \\
\mathrm{H}_{l} \\
\mathrm{E}\end{array}$ & $\begin{array}{l}1.9482 \\
0.3407 \\
2.2890\end{array}$ & $\begin{array}{l}1.7453 \\
0.3650 \\
2.1103\end{array}$ & $\begin{array}{l}1.6471 \\
0.3525 \\
2.0996\end{array}$ & $\begin{array}{l}1.8759 \\
0.3589 \\
2.2349\end{array}$ & $\begin{array}{l}1.7291 \\
0.3651 \\
2.0943\end{array}$ & $\begin{array}{l}1.6663 \\
0.3619 \\
\mathbf{2 . 0 2 8 2}\end{array}$ \\
\hline Fig. 4 & $\begin{array}{l}\mathrm{H}_{r} \\
\mathrm{H}_{l} \\
\mathrm{E}\end{array}$ & $\begin{array}{l}2.5946 \\
0.3484 \\
2.8430\end{array}$ & $\begin{array}{l}2.4186 \\
0.3086 \\
2.7272\end{array}$ & $\begin{array}{l}2.4405 \\
0.3315 \\
2.7721\end{array}$ & $\begin{array}{l}2.1537 \\
0.3393 \\
2.4931\end{array}$ & $\begin{array}{l}2.1319 \\
0.3082 \\
2.4402\end{array}$ & $\begin{array}{l}2.1141 \\
0.3236 \\
\mathbf{2 . 4 3 7 7}\end{array}$ \\
\hline
\end{tabular}

by all methods. Table 3 gives the partition coefficients, partition entropy, reconstruction error rate and segmentation accuracy rate obtained by different methods running on Berkeley Segmentation Database. The maximum of partition coefficient, minimum of partition entropy, minimum of reconstruction error rate and maximum of segmentation accuracy rate are all marked in bold type. It is obvious that the value obtained by this method is much better than other methods.

Table 4 shows the evaluation indicators based on entropy obtained by running the various methods on different images, where the minimum values are marked in bold. It can be seen from the table that the evaluation index value corresponding to the segmentation result obtained by the NKWNLICM method is the smallest, and these results fully show that the NKWNLICM method has a superior effect on noise images.

Both of visual results and quantitative comparison demonstrate the excellent segmentation performance obtained by the proposed NKWNLICM method. This result indicates that Neutrosophic C-means Clustering with local information and noise distancebased kernel metric is able to overcome the effect of noise on segmentation results and is good at maintaining image structure information.

\section{Conclusions}

In this paper, we proposed a new clustering algorithm, Neutrosophic C-means clustering with Local Information and noise distance-based Kernel Metric for Image segmentation (NKWNLICM), and applied it to the segmentation study of noise images. The objective function of NKWNLICM is convex to each variables, and the value of the objective function tends to be stable with the increase of iteration number. So we think it is convergence empirically. By introducing the concept of local fuzzy information and noise distance in the NCM algorithm, the algorithm does not need parameters setting for different noises when segmenting images, which makes the algorithm overcome the noise effect. The efficiency of the proposed method is evaluated on grayscale image segmentation applications. Experimental results show that the algorithm has better segmentation results for noisy images. In addition, we plan to apply the method to the more complex data in our future works. In the proposed method, the local information of image, distance of noise and kernel function are integrated for image segmentation modeling. Thus, its segmentation efficiency has little advantage over the other methods. In future, we will use parallel computing to improve its efficiency. 


\section{Acknowledgments}

This work has been supported in part by the National Natural Science Foundation of China (Grant Nos. 61773220, 61502206), the Nature Science Foundation of Jiangsu Province under Grant (No. BK20150523) and the Open Project Program of Key Laboratory of Jiangsu Key Laboratory of Meteorological Observation and Information Processing under grant number (KDXS1807).

\section{References}

[1] R. Hong, L. Zhang, C. Zhang, et al., Flickr circles: aesthetic tendency discovery by multi- view regularized topic modeling, IEEE Trans. Multimed. 18 (8) (2016) $1555-1567$.

[2] R. Hong, L. Zhang, D. Tao, Unified photo enhancement by discovering aesthetic communities from Flickr, IEEE Trans. Image Process. 25 (3) (2016) 1124-1135.

[3] R. Hong, Z. Hu, R. Wang, et al., Multi-view object retrieval via multi-scale topic models, IEEE Trans. Image Process. 25 (12) (2016) 5814-5827.

[4] S. Miyamoto, H. Ichihashi, K. Honda, Algorithms for fuzzy clustering, Studies Fuzziness Soft Comput. 229 (2008), https://doi.org/10.1007/978-3-540-787372.

[5] J. Bezdek, R. Hathaway, M. Sobin, Convergence theory for fuzzy c-means: counterexamples and repairs, IEEE Trans. Systems Man Cybernet. 17 (5) (1987) $873-877$.

[6] K. Chuang, S. Tzeng, H.L. Chen S, Fuzzy c-means clustering with spatial information for image segmentation, Comput. Med. Imaging Graph. 30 (1) (2006) 9.

[7] R. Dave, N Characterization and detection of noise in clustering, Pattern Recogn. 12 (11) (1991) 657-664.
[8] S. Krinidis, V. Chatzis, A robust fuzzy local information C-Means clustering algorithm, IEEE Trans. Image Process. A Publication IEEE Signal Process. Soc. 19 (5) (2010) 1328-1337.

[9] M. Gong, Y. Liang, J. Shi, Fuzzy C-means clustering with local information and kernel metric for image segmentation, IEEE Trans. Image Process. 22 (2) (2013) 573-584.

[10] Y. Cuo, H. Sengur A, NCM : neutrosophic C-means clustering algorithm, Pattern Recognition 488 (2015) 2710-2724.

[11] M. Jian, Q. Qi, J. Dong, Y. Yin, K.M. Lam, Integrating qdwd with pattern distinctness and local contrast for underwater saliency detection, J. Visual Commun. Image Represent. 53 (2018) 31-41.

[12] P. Jing, Y. Su, L. Nie, Low-rank multi-view embedding learning for micro-video popularity prediction, IEEE Trans. Knowl. Data Eng. PP (99) (2018) 1519-1532.

[13] L. Zhu, J. Shen, L. Xie, Z. Cheng, Unsupervised visual hashing with semantic assistant for content-based image retrieval, IEEE Trans. Knowledge Data Eng. 29 (2) (2017) 472-486.

[14] L. Zhu, Z. Huang, X. Liu, Discrete multimodal hashing with canonical views for robust mobile landmark search, IEEE Trans. Multimedia 19 (9) (2017) 2066 2079.

[15] P. Jing, Y. Su, L. Nie, A framework of joint low-rank and sparse regression for image memorability prediction, IEEE Trans. Circuits Syst. Video Technol. PP (99) (2018), 11.

[16] F. Smarandache, Neutrosophy: neutrosophic probability, set, and logic: analytic synthesis and synthetic analysis. Philosophy, Cambridge, 1998.

[17] H. Zhang, J. Fritts E, Entropy-based objective evaluation method for image segmentation, Procee. Spie 5307 (2003) 38-49.

[18] C. James, Bezdek $\dagger$, Cluster validity with fuzzy sets, J. Cybernet. 3 (3) (1973) $58-73$.

[19] T. Esch, M. Thiel, M. Bock, Improvement of image segmentation accuracy based on multiscale optimization procedure, IEEE Geosci. Remote Sens. Lett. 5 (3) (2008) 463-467.

[20] W. Pedrycz, Knowledge-Based Clustering: From Data to Information Granules, Wiley-Interscience, 2005. 